УДК 514.756

\title{
К геометрии бутылки Клейна
}

М.А. Чешкова

Алтайский государственный университет (Барнаул, Россия)

\section{To the Geometry of the Klein Bottle}

M.A. Cheshkova

Altai State University (Barnaul, Russia)

Данная публикация продолжает серию работ автора о моделировании односторонних поверхностей. На односторонней поверхности существует замкнутая кривая (дезориентирующий контур), обладающая тем свойством, что при еt обходе локальная ориентация в касательном пространстве меняет знак.

Односторонней поверхностью является бутылка Клейна.

Рассматриваются две гладкие вектор-функции. Предполагается, что одна из них есть $2 \pi$ периодическая, другая $2 \pi-$ антипериодическая.

С использованием найденных функций определяются уравнения бутылки Клейна, дезориентирующие контуры и уравнения двух листов Мебиуса, на которые разрезается бутылка Клейна. В работе исследуется инверсия бутылки Клейна.

Доказывается, что если бутылка Клейна не проходит через центр инверсии, то инверсия бутылки Клейна есть бутылка Клейна. Доказывается также, что если бутылка Клейна не проходит через центр инверсии, то дезориентирующие контуры бутылки Клейна при инверсии перейдут в дезориентирующие контуры.

С помощью системы компьютерной математики строятся исследуемые поверхности.

Ключевые слова: бутылка Клейна, лист Мебиуса, тор, периодическая функция.

\section{DOI 10.14258/izvasu(2019)4-18}

\section{Введение}

Если на поверхности в $E^{3}$ существует замкнутая кривая (дезориентирующий контур), обладающая тем свойством, что при ее обходе локальная ориентация в касательном пространстве меняет знак, то поверхность называется односторонней.

Впервые уравнение односторонней поверхности, открытой Мебиусом, было получено Машке [1]. Если гауссова кривизна листа Мебиуса равна нулю, то он называется плоским. Библиография работ на эту тему дана в работе [2].

К односторонним поверхностям относятся: скрещенный колпак [3, с. 304], римская поверхность [3, с. 305], [4, с. 302], поверхность Боя [3, с. 305], бутылка Клейна [3, с. 306]. Римская поверхность, поверх-
This paper continues the author's series of works on the modeling of one-sided surfaces. There is a closed curve (disorienting contour) on a one-sided surface, and this curve has the property of changing the sign when it is bypassed local orientation in tangent space.

A one-sided surface is a Klein bottle. Two smooth vector functions are considered. It is assumed that one of them is $2 p i$ - periodic, the other is $2 p i$ - antiperiodic. The Klein bottle equations, disorienting contours, and the equations of two Mobius sheets into which a Klein bottle is cut are determined using the obtained functions. In this paper, the inversion of the Klein bottle is investigated.

We prove that if a Klein bottle does not pass through the center of inversion, then the inversion of the Klein bottle is also the Klein bottle. It is also proved that if the Klein bottle does not pass through the center of inversion, then the disorienting contours of the Klein bottle under the inversion will pass into the disorienting contours. Models of the considered surfaces are built with the help of the computer mathematics system.

Key words: Klein bottle, Mobius strip, torus, periodic function.

ность Боя и скрещенный колпак с крышкой являются моделью проективной плоскости. Односторонние поверхности исследуются [5-13].

\section{1. Основной результат}

В евклидовом пространстве $E^{3}$ рассмотрим две гладкие вектор-функции $s=s(u), l=l(u), u \in[-\pi, \pi]$. Предполагается, что $s=s(u)$ есть $2 \pi$-периодическая, $l(u)$ есть $2 \pi$-антипериодическая функции, не равные нулю на этом промежутке, и векторное произведение $\left[s^{\prime}(u), l(u)\right] /=0$.

С помощью этих функций построим пример односторонней поверхности $K L$, которая является моделью бутылки Клейна, и исследуем ее. 
Поверхность $K L$ зададим параметрически в виде:

$$
\begin{aligned}
& r(u, v)=(p+\cos (v)) s(u)+\sin (v) l(u), p \neq \mp 1, \\
& u \in[-\pi, \pi], v \in[-\pi, \pi] .
\end{aligned}
$$

Замечание. В [7] исследуется поверхность $K$ :

$r(u, v)=s(u)+\sin (v) l(u) \pm \sin (k v)(l(u+\pi)+f(u) e)$

$u \in[\pi, \pi], v \in[-\pi, \pi], k \in N$, где $f=f(u)-2 \pi$ антипериодическая функция, а вектор $е$ есть постоянный.

Вектор $f(u) e$ удобно выбрать так, чтобы векторы $l(u), l(u+\pi)+f(u) e$ были ортогональными.

Если $k$ - четное число, то кривая $v=$ const есть кривая типа восьмерки с $k$ секциями и поверхность $K$ замкнутая. Поверхность $K$ определяет модель бутылки Клейна. Кривая $r=r(u, 0)$ есть дезориентирующий контур поверхности $K$.

Если $k \neq 1$ - нечетное число, то кривая $v=$ const есть незамкнутая кривая, а поверхность $K$ есть поверхность с краем.

При $k=1$ это отрезок прямой, и поверхность $K$ есть прямолинейный лист Мебиуса.

Исследуем поверхность $K L$.

Теорема 1. Поверхность $K L$ определяет модель бутылки Клейна, а кривые

$$
\begin{aligned}
& S_{1}: r(u, 0)=(p+1) s(u), \\
& S_{2}: r(u, \pi)=(p-1) s(u)
\end{aligned}
$$

- дезориентирующие контуры поверхности KL.

Доказательство. Рассмотрим бутылку Клейна как фактор-пространство $[7$, с. 75$]$

$K^{*}=[-\pi, \pi] \times[-\pi, \pi] /[(-\pi,-v) \sim$ $(\pi, v),(u,-\pi) \sim(u, \pi)]$.

Действительно, $r(u,-\pi)=(p-1) s(u)=r(u, \pi)$, $r(-\pi,-v)=(p+\cos (-v)) s(-\pi)+\sin (-v) l(-\pi)=$ $(p+\cos (v)) s(\pi)-\sin (-v) l(\pi)=r(\pi, v)$.

Рассмотрим замкнутую кривую $S_{1}: r(u, 0)=$ $(p+1) s(u)$.

Имеем

$$
r_{1}=r_{u}(u, 0)=(p+1) s^{\prime}(u), r_{2}=r_{v}(u, 0)=l(u) .
$$

Касательное пространство в точках кривой $S_{1}: r(u, 0)=(p+1) s(u)$ примет вид

$$
T_{m} K L=\left\{l(u), s^{\prime}(u)\right\}, m \subset S_{1} .
$$

Замечаем, что базисы

$$
\begin{gathered}
\left.\left\{r_{1}(u), r_{2}(u)\right\}=\{l(u)), s^{\prime}(u)\right\}, \\
\left\{r_{1}(u+2 \pi), r_{2}(u+2 \pi)\right\}=\left\{l(u+2 \pi), s^{\prime}(u+2 \pi)\right\}
\end{gathered}
$$

противоположно ориентированы. Таким образом, кривая $S_{1}: r(u, 0)=(p+1) s(u)$ есть дезориентирующий контур.
Аналогично проверяется, что кривая $S_{2}$ : $r(u, \pi)=(p-1) s(u)$ есть дезориентирующий контур.

Таким образом, $S_{1}, S_{2}$ - дезориентирующие контуры поверхности $K L$.

Разрежем $K L$ вдоль кривой $r=r\left(u, v_{0}\right), u \in$ $[-2 \pi, 2 \pi], v_{0} \neq 0, \mp \pi$. Получим два листа Мебиуса (криволинейных) со средними линиями $r(u, 0)=$ $(p+1) s(u), r(u, \pi)=(p-1) s(u)$.

Если $p+1=0(p-1=0)$, то средняя линия $r=(p+1) s(u)(r=(p-1) s(u))$ вырождается в точку. Один из листов Мебиуса вырождается в конус, гомеоморфный сфере с дырой. Поверхность в этом случае гомеоморфна сфере с дырой, заклеенной листом Мебиуса. Имеем модель проективной плоскости [14, с. 25].

Рассмотрим инверсию [15, с. 482]

$$
r *=\frac{m^{2} r}{<r, r>}
$$

относительно сферы радиуса $m$ с центром в начале координат.

Теорема 2. Если бутылка Клейна KL не проходит через иентр инверсии, то инверсия бутылки Клейна KL есть бутылка Клейна.

Доказательство. Пусть функция $r=r(u, v), u \in[-\pi, \pi], v \in[-\pi, \pi]$ определяет модель бутылки Клейна. Склейка прямоугольника определяется равенствами $r(u,-\pi)=r(u, \pi)$, $r(-\pi,-v)=r(\pi, v)$.

Рассмотрим функцию $\psi=<r, r>$. По условию теоремы $\psi \neq 0$ на поверхности.

Нетрудно проверить, что $\psi(\pi, v)=\psi(-\pi,-v)$, $\psi(u,-\pi)=\psi(u, \pi)$.

Имеем $r^{*}(u,-\pi)=r^{*}(u, \pi), r^{*}(-\pi,-v)=$ $r^{*}(\pi, v)$. Поверхность $r^{*}=r^{*}(u, v)$ есть модель бутылки Клейна.

Обозначим поверхность, которая получается из поверхности $K L$, путем инверсии (4) через $K L I$.

Теорема 3. Если бутылка Клейна KL не проходит через иентр инверсии, то дезориентирующие контурь поверхности KL при инверсии (4) перейдут в дезориентирующие контуры поверхности KLI.

Доказательство. Имеем $r(u, 0)=(p+$ 1) $s(u),<\quad r\left(u, 0 \quad>, r\left(u, 0 \quad>=(p+1)^{2}<\right.\right.$ $s(u), s(u)>\neq 0$,

$$
\begin{gathered}
r_{u}(u, 0)=(p+1) s^{\prime}(u), r_{v}(u, 0)=l(u), \\
r_{u}^{*}(u, 0)=m^{2}\left(\frac{-2<s^{\prime}(u), s(u)>s(u)}{(p+1)<s(u), s(u)>^{2}}+\right. \\
\left.\frac{s^{\prime}(u)}{(p+1)<s(u), s(u)>}\right), \\
r_{v}^{*}(u, 0)=m^{2}\left(\frac{-2<s(u), l(u)>s(u)}{(p+1)^{2}<s(u), s(u)>^{2}}+\right.
\end{gathered}
$$




$$
\left.\frac{l(u)}{(p+1)^{2}<s(u), s(u)>}\right) .
$$

Так как $r_{u}^{*}(u, 0)=r_{u}^{*}(u+2 \pi), r_{v}^{*}(u, 0)=-r_{v}^{*}(u+$ $2 \pi)$, то кривая $r=r^{*}(u, 0)$ есть дезориентирующий контур поверхности $K L I: r=r^{*}(u, v)$.

Аналогично проверяется, что кривая $r=$ $r^{*}(u, \pi)$ есть дезориентирующий контур поверхности $K L I: r=r^{*}(u, v)$.

Дезориентирующие контуры поверхности $K L I: r=r^{*}(u, v)$ имеют вид

$$
\begin{aligned}
& S_{1}^{*}: r^{*}(u, 0)=\frac{m^{2} s(u)}{(p+1)<s(u), s(u)>}, \\
& S_{2}^{*}: r^{*}(u, \pi)=\frac{m^{2} s(u)}{(p-1)<s(u), s(u)>} .
\end{aligned}
$$

Сравним $S_{1}, S_{1}^{*}$ и $S_{2}, S_{2}^{*}$.

Из формул (2),(3),(5), (6) вытекает следующее утверждение.

Теорема 4. Если длина вектора $s(u)$ постоянная и $m=(p+1)|s(u)|$, то кривые $S_{1}, S_{1}^{*}$ совпадают. Если $m=(p-1)|s(u)|$, то совпадают кривые $S_{2}, S_{2}^{*}$.

\section{2. Пример бутылки Клейна $K L$}

Рассмотрим гладкую замкнутую неплоскую кривую $\gamma$, заданную $4 \pi$-периодической векторфункцией $\rho=\rho(u)$, которая не является $2 \pi-$ периодической и $2 \pi$ - антипериодической.

Так как $\rho(u)=\rho(u+4 \pi)$, то функция

$$
s(u)=\frac{1}{2}(\rho(u)+\rho(u+2 \pi))
$$

есть $2 \pi$-периодическая не равная нулю, а векторфункция

$$
l(u)=\frac{1}{2}(\rho(u)-\rho(u+2 \pi))
$$

есть $2 \pi$-антипериодическая не равная нулю.

Рассмотрим тор в $E^{3}$

$r(u, v)=$

$((a+b \cos (v)) \cos (u),(a+b \cos (v)) \sin (u), b \sin (v))$.

Зададим линию $v=k u / 2$, где $k$ нечетное число.

Тогда вектор-функция

$$
\begin{gathered}
\rho(u)=((a+b \cos (k u / 2)) \cos (u), \\
(a+b \cos (k u / 2) \sin (u), b \sin (k u / 2))
\end{gathered}
$$

$u \in[-2 \pi, 2 \pi]$ есть $4 \pi$-периодическая (обмотка тоpa).

Рассмотрим тор и кривую на нем при $a=2$, $b=1, k=1$.

Имеем

$$
s(u)=(2 \cos (u), 2 \sin (u), 0),
$$

$l(u)=(\cos (u) \cos (u / 2), \sin (u) \cos (u / 2), \sin (u / 2))$.

Бутылку Клейна $K L$ зададим в виде

$$
\begin{gathered}
r=(4+\cos (v)) 2 \cos (u)+\sin (v) \cos (u) \cos (u / 2) \\
(4+\cos (v)) 2 \sin (u)+\sin (v) \sin (u) \cos (u / 2), \\
\sin (v) \sin (u / 2)), u \in[-\pi, \pi,], v \in[-\pi, \pi] .
\end{gathered}
$$

Построим обмотку тора и бутылку Клейна $K L$ (рис. 1).
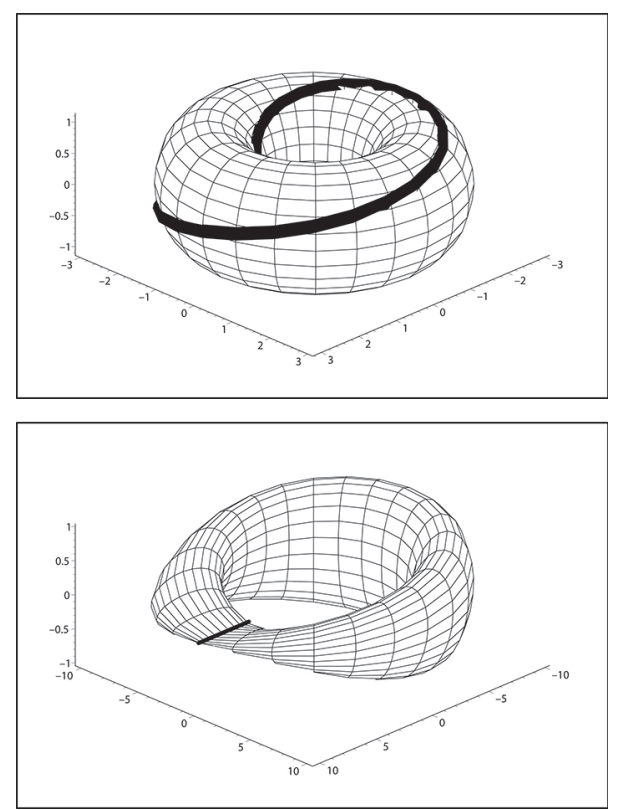

Рис. 1. Обмотка тора и бутылка Клейна $K L$

Бутылка Клейна $K L$ имеет самопересечение: прямая

$$
r=r(0, v)=((4+\cos (v)) 2+\sin (v))(1,0,0) .
$$

При инверсии (4) она переходит в прямую

$$
r=r^{*}(0, v)=\frac{m^{2}}{(4+\cos (v)) 2+\sin (v)}(1,0,0),
$$

которая является прямой самопересечения бутылки Клейна $K L I$.

Кроме того, будем строить кривую (эллипс) $\kappa$ : $r=r(\pi / 2, v)=((4+\cos (v))(-2,0,0)+\sin (v)(0,0,1)$ и ее инверсию $\kappa^{*}$.

Так как $p=4,|s(u)|=2$, то рассмотрим различные случаи $m=10, m=6, m \neq 10,6$.

2.1. Случай $m=10$.

Построим бутылку Клейна $K L$ (светлая) и ее инверсию $K L I$ (темная), а также кривые $\kappa$ (светлая) и $\kappa^{*}$ (темная) (рис. 2).

и $\kappa^{*}$ (темная) (рис. 3)

2.2. Случай $m=6$.

Построим бутылку Клейна $K L$ (светлая) и ее инверсию $K L I$ (темная) (рис. 4), а также кривые $\kappa$ (светлая) и $\kappa^{*}$ (темная) (рис. 5$)$. 


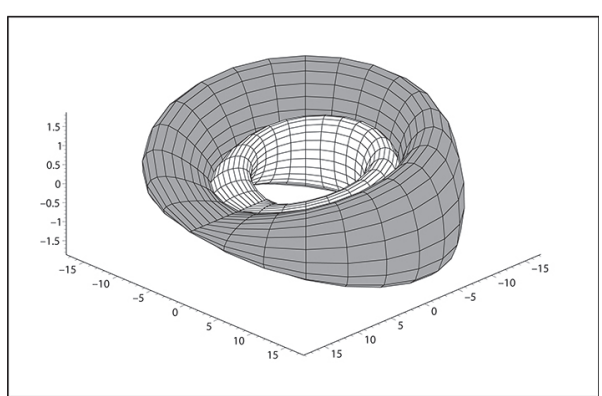

Рис. 2. Поверхности $K L, K L I$, кривые $\kappa, \kappa^{*} m=10$

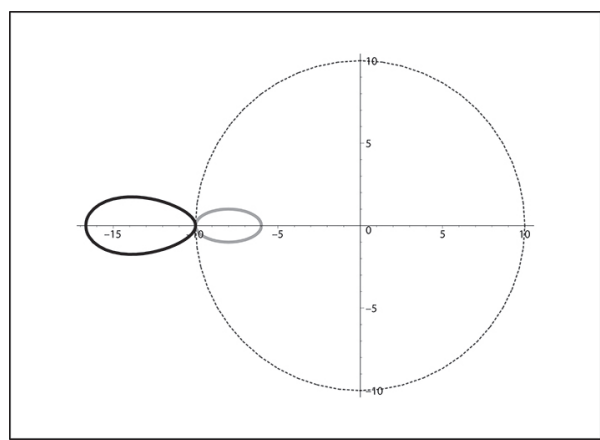

Рис. 3. Кривые $\kappa, \kappa^{*}, m=10$

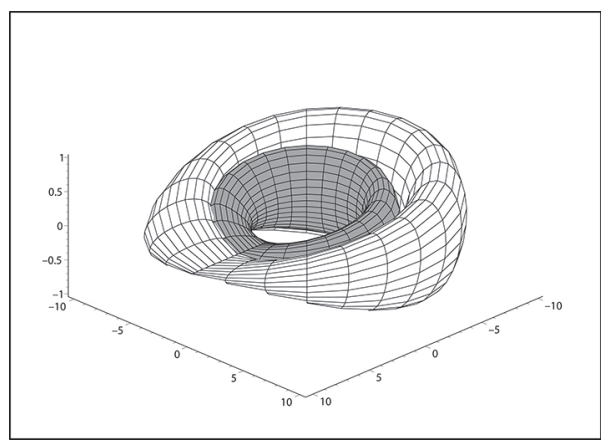

Рис. 4. Поверхности $K L, K L I, m=6$

\section{3. Случай $m=4$.}

Построим бутылку Клейна $K L$ (светлая) и ее инверсию $K L I$ (темная) (рис. 6), а также кривые $\kappa$ (светлая) и $\kappa^{*}$ (темная) (рис. 7$)$.

\section{4. Случай $m=12$.}

Построим бутылку Клейна $K L$ (светлая) и ее инверсию $K L I$ (темная), а также кривые $\kappa$ (светлая) и $\kappa^{*}$ (темная) (рис. 8).

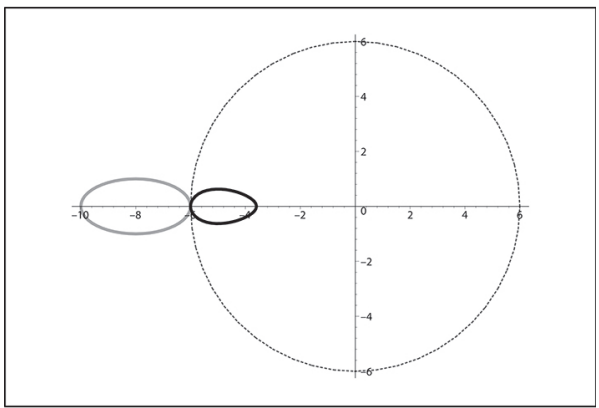

Рис. 5. Кривые $\kappa, \kappa^{*}, m=6$

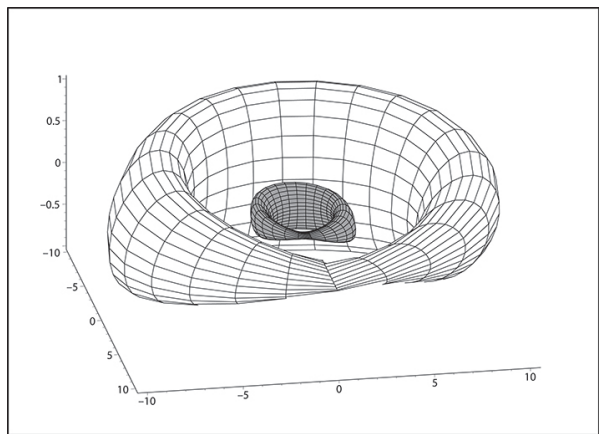

Рис. 6. Поверхности $K L, K L I, m=4$

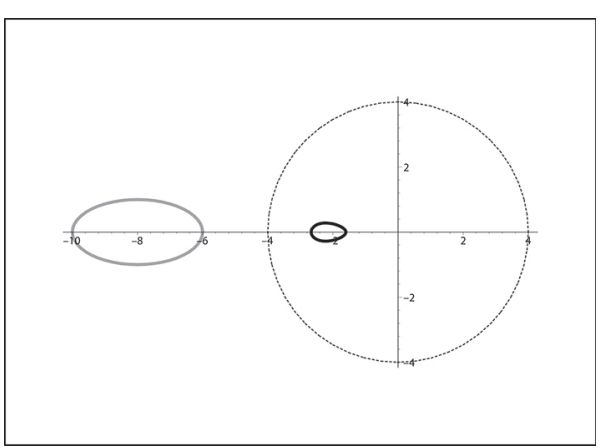

Рис. 7. Кривые $\kappa, \kappa^{*}, m=4$
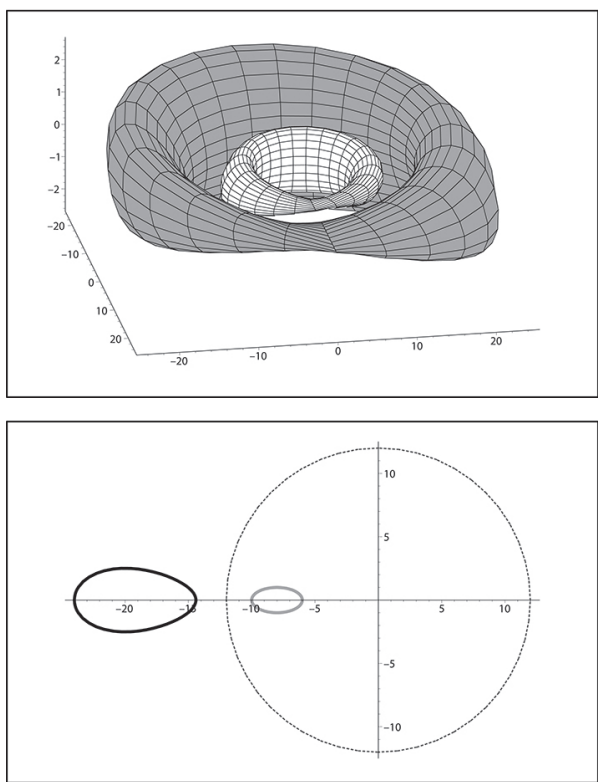

Рис. 8. Поверхности $K L, K L I$, кривые $\kappa, \kappa^{*}, m=12$ 
Заключение. Основным результатом работы является построение модели бутылки Клейна. На- ходятся дезориентирующие контуры. Исследуется инверсия бутылки Клейна.

\section{Библиографический список}

1. Mashke H. Note on the unilateral surface of Moebius // Trans.Amer.Math.Sos., 1:1 (1900).

2. Сабитов И.Х. Изометрические погружения и вложения плоского листа Мебиуса в евклидовы пространства // Известия РАН. 2007. Т. 71. № 5.

3. Кривошапко С.Н., Иванов В.Н., Халаби С.М. Аналитические поверхности. М., 2006.

4. Гильберт Д., Кон-Фоссен С. Наглядная геометрия. M., 1981.

5. Чешкова М.А. Об одной модели бутылки Клейна // Известия Алт. гос. ун-та. 2016. № 1 (89).

6. Чешкова М.А. Односторонние поверхности // Известия Алт. гос. ун-та. 2015. № 1/2 (85).

7. Чешкова М.А. Пример инверсии бутылки Клейна // Труды семинара по геометрии и математическому моделированию. Барнаул, 2016. № 2.

8. Cirilo-Lombaeeto D.I Coherent states for a quantum particle on Mobius // Письма в журнал «Физика элементарных частиц и атомного ядра». 2009. Т. 6 . № 5.
9. Словеснов А.В. Ленты Мебиуса с плоской метрикой // Вестник Моск. ун-та. Серия 1: Математика. 2009. № 5.

10. Шалагинов М.Ю., Иванов М.Г., Долгополов М.В. Задачи с оператором Лапласа на топологических поверхностях // Вестник Самарского гос. тех. ун-та. Серия : Физмат. науки. 2011. № 2 (23).

11. Борисюк А.Р. Глобальные бифуркации на бутылке Клейна. Общий случай // Математический сборник. 2015. Т. 196. № 4.

12. Набеева Л.Р. Классификация узлов в утолщенной бутылке Клейна. // Вестник Челяб. гос. ун-та. 2012. № 26 (280).

13. Карпухин М.А. Немаксимальность экстремальных метрик на торе и бутылке Клейна // Математический сборник. 2013. Т. 204. № 12.

14. Борисович Ю.Г., Близняков Н.М., Израилевич Я.А., Фоменко Т.Н. Введение в топологию. М., 1995.

15. Розенфельд Б.А. Многомерные пространства. М., 1966. 